\title{
Sticky stuff: xerostomia in patients undergoing head and neck radiotherapy-prevalence, prevention, and palliative care
}

\author{
James William Snider III' ${ }^{1}$, Charles Christian Paine II $^{2}$ \\ ${ }^{1}$ Department of Radiation Oncology, University of Alabama at Birmingham, UAB Proton Center, Birmingham, AL, USA; ${ }^{2}$ Department of Pediatrics/ \\ Palliative Medicine, University of Mississippi School of Medicine, University Hospital, Jackson, MS, USA \\ Contributions: (I) Conception and design: All authors; (II) Administrative support: All authors; (III) Provision of study materials or patients: All \\ authors; (IV) Collection and assembly of data: All authors; (V) Data analysis and interpretation: All authors; (VI) Manuscript writing: All authors; (VII) \\ Final approval of manuscript: All authors. \\ Correspondence to: James William Snider III, MD. Assistant Professor, Department of Radiation Oncology, University of Alabama at Birmingham, \\ UAB Proton Center, 400 20th Street S, Birmingham, AL 35233, USA. Email: jwsnider@uabmc.edu.
}

\begin{abstract}
Xerostomia remains one of the most common and impactful side effects associated with radiotherapy for head and neck malignancies. With improvements in oncologic therapy and an improved prognosis for patients with head and neck squamous cell carcinoma (HNSCC) overall, the long-standing effects of therapy have become even more significant. Recent efforts have primarily focused on prevention of this morbidity through technological advances, reductions in radiotherapy fields, and radioprotectants. The promises of de-intensified strategies and proton radiation warrant ongoing investigation. However, palliative care for and active management of xerostomia before, during, and after treatment deserve further attention and research to define optimal approaches.
\end{abstract}

Keywords: Radiation; palliative care; proton therapy; xerostomia; de-intensification; supportive care

Submitted Sep 15, 2019. Accepted for publication Feb 06, 2020.

doi: 10.21037/apm.2020.02.36

View this article at: http://dx.doi.org/10.21037/apm.2020.02.36

\section{Introduction}

Head and neck cancers of the oral cavity, pharynx, and larynx are estimated to be diagnosed in 65,410 new cases and to cause 14,620 deaths in the United States in 2019 (1). It is also the sixth most common cancer worldwide $(2,3)$. Head and neck squamous cell carcinoma (HNSCC), by far, remains the most common histology. There has been relative stability in the rates of diagnosis of head and neck cancers in the U.S. in recent years (4).

This relative stability has been attributed to two inverse shifts in causation of disease. As tobacco use, smoking and heavy alcohol abuse have gradually decreased in the U.S., a consequent decrease in laryngeal, oral cavity, and oropharyngeal cancers caused by these carcinogens has followed. However, this has not resulted in an overall decrease in the disease, as there has been a simultaneous increase in the incidence of human papilloma virusmediated squamous cell carcinoma (HPV-OPSCC) especially in the oropharynx. Luckily, the prognosis associated with HPV-OPSCC is much better than that of non-virally induced cancers, as long as appropriate management and oncologic therapy is undertaken $(5,6)$. A recursive partitioning analysis from a large randomized study of concurrent chemoradiotherapy for OPSCC proved the substantial prognostic importance of HPV-positivity in this disease entity.

Definitive management of these malignancies often involves a combination of surgical resection, radiotherapy, and systemic therapy (7). Radiotherapy is utilized as a curative or palliative modality for local control of disease in the head and neck region. It can often be utilized without surgical resection, as an organ preservation strategy, or in the adjuvant setting following resection to reduce the 

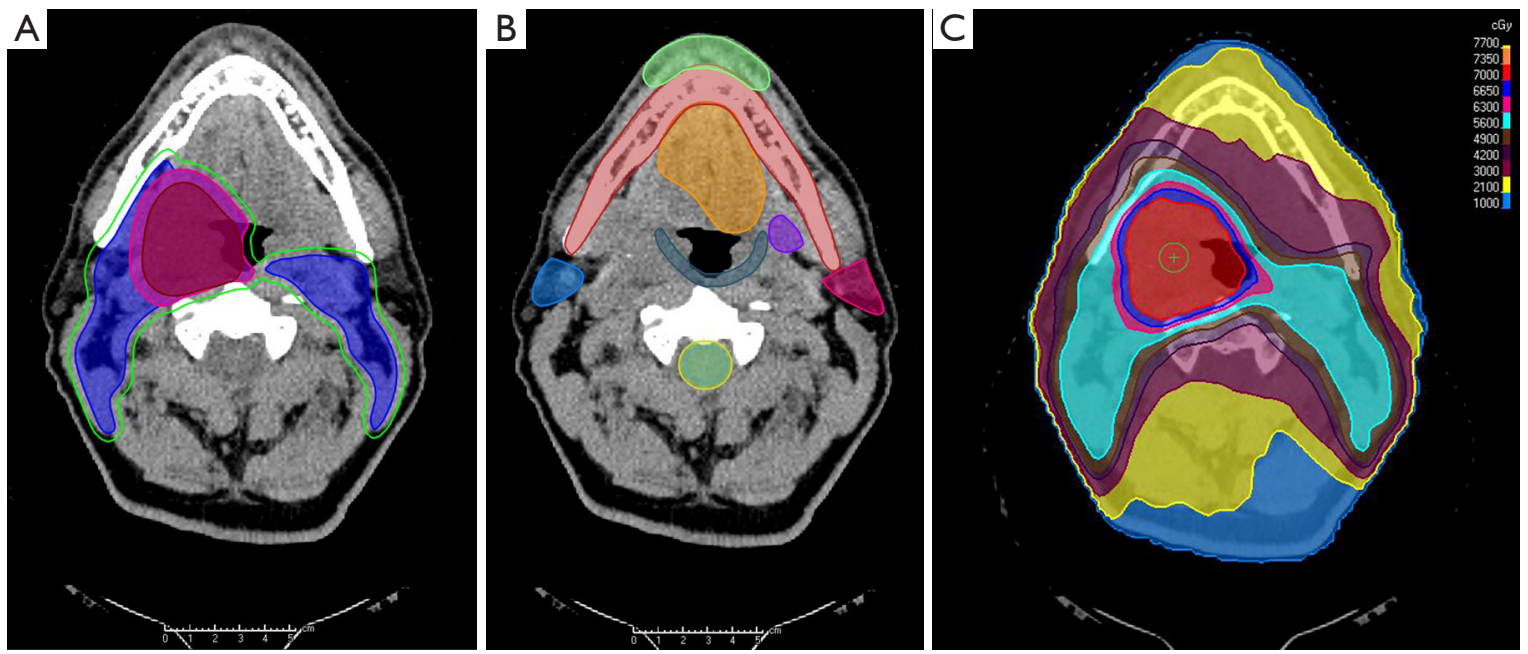

Figure 1 Example radiotherapy target volumes (A), organs at risk (B), and resultant volumetric modulated arc therapy treatment plan (C) for a patient with head and neck squamous cell carcinoma with primary disease in the right tonsil.

chance of recurrence in cases at risk. Radiotherapy in this region is generally delivered with standard or altered fractionation strategies over a five- to eight-week course of daily or twice daily treatments.

Though radiotherapy holds the promise of high rates of disease cure, particularly considering the relatively low rates of distant metastasis in HNSCC, it comes at a cost as many of the normal tissues in the head and neck region are particularly radiosensitive. Acute, subacute, and late morbidity associated with head and neck radiotherapy is undeniably substantial. Patients generally suffer from, at least, therapy-induced dysgeusia/altered taste, skin hyperpigmentation/desquamation, dysphagia, odynophagia, nausea/vomiting, alopecia, decreased oral intake, nutritional deficiency, feeding tube dependency, pain, trismus, and xerostomia.

Of these, xerostomia can be one of the most significant, lifelong side effects. Xerostomia can have tremendous effects on diet, nutrition, weight loss or gain, and quality of life well after completion of active therapy. With the aforementioned shift in this disease entity towards HPVOPSCC, as well as significant improvements in oncologic therapy and supportive care, an ever-increasing number of patients are surviving their malignancy but suffering from the long-term effects of oncologic therapy. An increased attention to addressing the side effects associated with therapy should follow. We will herein discuss the scope of the problem of xerostomia associated with oncologic therapy especially for HNSCC. We will subsequently address current strategies for lessening the impact of oncologic therapy on salivary flow rates, palliative options for mitigating the symptoms post-therapy, and future directions for research in improving xerostomia outcomes in this patient population.

\section{Mechanism of xerostomia in patients undergoing oncologic therapy for HNSCC}

Xerostomia is defined as the "subjective sensation of a dry mouth characterized by a marked decrease and/or thickening of the saliva" (8). This outcome, following head and neck radiotherapy in particular, stems from hypofunction of the major and minor salivary glands. The parotid glands provide the majority of stimulated salivary flow during eating and speech, and they are primarily supported by the other major salivary glands in this setting (9). However, this balance in function vastly shifts in unstimulated flow where the parotids contribute only approximately $20 \%$, the submandibular glands $65 \%$, sublingual $<10 \%$, and the minor salivary glands $<10 \%$.

Radiotherapy to the head and neck region for HNSCC most commonly involves targeting of the primary site as well as bilateral draining neck lymphatics. Figure 1 demonstrates a standard head and neck radiotherapy plan for a patient with OPSCC utilizing a conformal intensity modulated radiation therapy (IMRT) technique known as volumetric modulated arc therapy (VMAT). Figure $1 A$ demonstrates the "contouring" and delineation of the radiotherapy 
targets. Figure $1 B$ shows the associated, coplanar normal tissues which are contoured for dose delivery optimization. Figure $1 C$ illustrates the final, resultant VMAT radiotherapy plan in a simultaneous integrated boost (SIB) technique.

Dependent upon the primary disease site, extent, and clinically/radiographically appreciated nodal involvement at diagnosis, certain levels of the neck lymphatics will be covered at least to moderate radiotherapy doses to sterilize any microscopic involvement. While this is custom-tailored to the individual clinical scenario in all cases, level II (upper jugular region) and level $\mathrm{Ib}$ (submandibular region) nodes of the neck lie in close proximity and abut/encompass the parotid glands and submandibular glands, respectively. Level II is the most commonly targeted as it acts as a central hub for lymphatic drainage from most of the subsites of the head and neck region (10). Level Ib is commonly targeted for oral cavity and sinonasal primary disease as well as in the case of bulky level II adenopathy in the ipsilateral neck. Bulky adenopathy especially in level II can cause retrograde flow into level Ib nodes from sites that generally drain primarily to the jugular chain.

As a result of the juxtaposition of the primary disease as well as these draining lymphatics, the major and minor salivary glands are invariably affected by radiotherapy for HNSCC. They are exposed to the moderate to low doses of radiation that result from entrance and exit dose with photon/X-ray techniques for delivery. The role of particle therapies in reducing dose to critical structures will be discussed later. As expected, these particularly radiosensitive structures demonstrate early and late sequela as a result of this exposure.

The specific mechanisms for diminution in salivary flow as a result of radiation exposure remain somewhat controversial, but several models have been at least partially validated. The dramatic acute and subacute diminutions in salivary flow during and immediately following radiotherapy generally violate the sense of how normal tissues of the body should respond to radiotherapy (11). As the excretory cells that supply salivary function are well-differentiated and should have slow mitotic rates and resultant cell turnover, in theory, xerostomia should be a late complication at best, and the salivary glands should be relatively radio-insensitive. Obviously, this is not the case with virtually all patients showing symptomatology.

Initially, it was proposed that significant apoptosis may lead to the leakage of granules and lysis of acinar cells, but cell loss during early periods in radiotherapy has been invalidated in animal models (12). Instead, the acute and subacute xerostomia induced by radiotherapy is theorized to stem from damage to the plasma membrane of acinar cells. This seems to diminish water excretion by downregulating receptor-mediated signaling. The late persistence of xerostomia likely results from traditional cell killing of progenitor stem cells within the salivary glands. The transition from the former mechanism to the latter may explain the trend towards some improvement in xerostomia between 6-18 months post-treatment, followed by general permanence of the complication (13-15). The combination of the early and late effects fits most with the timeline of xerostomia throughout and after a course of head and neck radiotherapy.

\section{Prevalence and impact of xerostomia in patients undergoing oncologic therapy for HNSCC}

A systematic review by the Multinational Association of Supportive Care in Cancer and the International Society of Oral Oncology estimated, based on the extensive trials to date, the prevalence of xerostomia during and after the course of radiotherapy $(16,17)$. This effort served to clarify the time course of xerostomia from start of radiotherapy to diminution in function as well as the extent of recovery and long-term compromise. Xerostomia was present in very few patients at baseline (6-12\%), but nearly all patients (81$100 \%$ ), regardless of radiotherapy technique, experienced clinically significant xerostomia during the radiotherapy treatment course. The vast majority of patients reported or were objectively found to have xerostomia at 1-3 months, 3-6 months, 6-12 months, $1-2$ years, and $>2$ years posttreatment.

With xerostomia then representing one of the most common acute, subacute, and late side effects associated with head and neck radiotherapy, its impact on patients' overall function and quality of life can be profound. Xerostomia itself can be disturbing to a patient, but its downstream effects are arguably more significant. Diminution in salivary function involves changes in volume, consistency, and $\mathrm{pH}$ of secretions intraorally (18). These intraoral changes have been linked to dysgeusia, intraoral pain, swallowing dysfunction/dysphagia/odynophagia, difficulty in speech, demineralization of teeth, increased intraoral cariogenic flora, increased dental caries, overall fatigue, malnutrition, weight loss, osteoradionecrosis, reduced work/functional capacity, and worsened overall quality of life $(13,19-33)$.

Considering xerostomia's centrality to many of the 
acute and long-term morbidities associated with the treatment of HNSCC, it comes as no surprise that this symptom plays a key role in a number of quality-of-life metrics for treated patients. The University of Washington Quality of Life Questionnaire (UW-QOL), the M.D. Anderson Dysphagia Inventory (MDADI), the European Organization for Research and Treatment of Cancer Quality of Life Questionnaire Head and Neck 35 (EORTC QLQ-H\&N35), and the EORTC QLQ Core 30 (EORTC QLQ-C30), to name a few, all prominently feature either salivary flow directly or linked downstream effects in swallowing, taste, or speech (34). A recent systematic review and meta-analysis tracked changes in these metrics across 1,366 patients treated for OPSCC and attempted to estimate the impact of treatment on overall quality of life. This review found the most significant deteriorations in quality of life stemmed from the following in each questionnaire: UW-QOL-saliva, chewing, swallowing, speech, taste, appearance, and shoulder; MDADI—physical, global, emotional; EORTC QLQ-H\&N35-dry mouth, sticky saliva.

Without a doubt, treatment-related xerostomia plays an important role in patients' overall function, experience of radiation-associated morbidity, and long-term quality of life. Its impact should not be underestimated by the oncology and palliative care teams alike. In recent years, there have been a number of developments in both the prevention and treatment of radiation-associated xerostomia in patients with HNSCC. We will summarize these in the sections that follow.

\section{Techniques for prevention of radiation-induced xerostomia}

Prevention of radiation-associated xerostomia would clearly be the best method for reducing this morbidity's impact on patients' quality of life following oncologic therapy. Xerostomia is a deterministic, non-stochastic, effect of radiation exposure. A threshold of radiotherapy dose exists below which damage to the relevant salivary glands can be reduced, if not prevented. Clinically validated dose constraints to the salivary-associated organs at risk (parotid glands, submandibular glands, and oral cavity) have been established in recent years.

In the era of 2-dimensional radiotherapy delivery, little parotid or submandibular sparing was technically feasible. HNSCC was most commonly treated with opposed lateral fields to cover the primary site and upper neck. These fields were delineated by the treated radiation oncologist on a lateral simulation $\mathrm{X}$-ray. There was no organ-at-risk (OAR) delineation, nor dose-volume calculations. The parotid and submandibular glands commonly lay completely within the field of radiotherapy due to their juxtaposition on either side of the head, abutting the oropharynx and posterior oral cavity-common sites of primary disease. With the transition to 3-dimensional techniques, the treating radiation oncologist finally had a CT simulation scan on which OARs could be contoured and doses estimated in a volumetric fashion. While the technique and beam arrangement for HNSCC radiotherapy remained largely the same, a better understanding of dose thresholds for dysfunction of OARs was established.

With the advent of IMRT, oncologists were able to leverage an inverse planning algorithm and numerous inhomogeneous radiotherapy fields against the issue of salivary gland dose. IMRT allows several radiotherapy fields around the patient to be arranged so that each delivers an inhomogeneous part of the entire homogeneous dose expected across the target. Sophisticated computer algorithms were developed to calculate iteratively an optimal "solution" for delivering the desired radiotherapy dose to the target while maximally sparing OARs. VMAT represents a more extreme example of this, vastly increasing the number of beams or segments as the linear accelerator "arcs" around the patient.

The parotid glands were technically and feasibly the easiest glands to spare, and quickly clinical experiences validated certain thresholds and metrics which would provide for meaningful sparing of a salivary gland. For the parotids, less than a mean of 26 Gy to the entire gland or a V30 (volume receiving $30 \mathrm{~Gy}$ ) less than $50 \%$ were established as goals for parotid-sparing IMRT (21,35-38). Similar studies were completed for the submandibular glands which have established a goal mean of less than 39 Gy for preservation of serviceable gland function (39).

Three randomized controlled trials were run to compare IMRT to $2 \mathrm{D}$ and $3 \mathrm{D}$ techniques in relation to salivary function preservation and resultant associated morbidities. The study by Pow et al. randomized 51 patients to IMRT versus conventional radiotherapy for nasopharyngeal cancer. With conventional radiotherapy, only $4.8 \%$ and $9.5 \%$ of patients had regained at least $25 \%$ of their pre-radiotherapy stimulated-whole and stimulated-parotid salivary flow at 1-year post-treatment; patients treated with IMRT regained these functions at much higher rates of $50 \%$ and $83.3 \%$, respectively (40). In a similar study at the Hong 
Kong Cancer Institute, 60 patients with nasopharyngeal cancer were randomized (41). Observer rated xerostomia for these patients at 1 year differed substantially from $2 \mathrm{D}$ to IMRT, $82.1 \%$ versus $39.3 \%$. Stimulated, parotid and whole, salivary flow rates were also measurably higher in patients treated with IMRT. Finally, a multi-institutional study performed in the UK randomized 94 patients to IMRT versus conventional radiotherapy (42). Grade 2 xerostomia at 1 year was substantially lessened from $74 \%$ to $38 \%$ with IMRT. At 2 years, the gap widened to $83 \%$ versus $29 \%$. At both 12 and 24 months, recovery of salivary flow, drymouth-specific quality of life, and global quality of life were all significantly improved with IMRT. None of these studied found substantial differences in oncologic outcomes. Consequently, IMRT became the standard of care for head and neck radiotherapy and remains as such to date.

In an effort to improve the application of IMRT to head and neck radiotherapy, cooperative group efforts have further centered around standardization of both target and OAR delineation. Grégoire et al. have published guidelines for both primary clinical target volumes and delineation of neck nodal levels which guide radiation oncologists worldwide to date $(10,43)$. Additionally, consensus OAR contouring guidelines have helped ensure that dosimetric criteria utilized for sparing of OARs are properly applied to a well-delineated organ (44). Without this uniformity, doseconstraints remain of little value or prognostic importance.

Current efforts to improve radiotherapy planning, with a goal of preventing/lessening resultant xerostomia, center around field reduction, particle therapy, dose de-escalation, and the use of surgical management as a part of definitive therapy. Field reduction, in particular, involves lessening the margins utilized for planning uncertainty and the removal of particular levels of prophylactic neck coverage. For instance, the rate of level $\mathrm{Ib}$ nodal involvement, especially on the contralateral side, has been repeatedly examined in nonoral cavity primaries. Lee et al. reported on 102 ipsilateral submandibular nodal dissections performed in OPSCC patients with radiographically negative level Ib regions (45). They found that only $4.3 \%$ of patients had ipsilateral pathologically positive Ib nodes (5.3\% in HPV-OPSCC), lending credence to the sense that the Ib nodal region, and thus the submandibular gland, could be spared in most cases except those with extensive nodal involvement. This particularly challenges uniform coverage of contralateral level Ib without contralateral level II involvement. Similarly, consensus guidelines for the treatment of nasopharyngeal carcinomas have begun to recommend standard omission of level $\mathrm{Ib}$ from the radiotherapy targets unless felt to be at particular risk as a result of clear involvement, substantial bulk to level II adenopathy, or extracapsular extension on other nodes (46). Others have attempted submandibular gland sparing when treating level Ib disease using IMRT, as failures medial to the gland are not frequently seen (47).

Additionally, there has been extensive research and validation of ipsilateral-only neck treatment in patients with well-lateralized tonsillar OPSCC. Huang et al. (48) updated a previous series by O'Sullivan et al. (49) demonstrating the safety of treating only the ipsilateral neck in early stage or limited, locally advanced tonsillar cancer. Intuitively, this approach substantially limits dose to contralateral salivary glands and should vastly improve xerostomia outcomes when applied appropriately.

Particle therapies, such as proton therapy or carbon ion therapy, have also been proposed to reduce the burden of xerostomia in patients following chemoradiotherapy for HNSCC. In particular, a large randomized study, initiated out of the M.D. Anderson Cancer Center as a phase II/ III multi-institutional effort, is currently evaluating this strategy based on preliminary results from case-matched and dosimetric analyses (50-52). Particle therapies are especially able to reduce moderate to low dose exposure to the oral cavity (minor salivary glands), submandibular glands, and parotid glands with similar target coverage based on the physical properties of the Bragg peak energy deposition of these approaches (Figure 2). Patient-reported outcomes are particularly prioritized in these studies to measure both the subjective changes in endpoints like xerostomia as well as their quality-of-life impacts. However, objective measurement of salivary flow is also included.

Due to improved oncologic outcomes in HPV-OPSCC, it has been further theorized that lower prescription radiotherapy doses could be utilized without sacrificing in cure rates. Several institutional and multi-institutional studies have furthered this belief and proposed that at least the standard prescription could be lowered from 70 to $60 \mathrm{~Gy}$ with de-intensified chemotherapeutic regimens (53). This theory has formed the basis of a large, multi-institutional cooperative group trial in NRG HN002 which is testing two experimental protocols for reduced dose chemoradiation or altered fractionation radiotherapy alone in HPV-OPSCC.

Alternatively, many clinicians and investigators have favored a primary surgical approach to OPSCC with transoral robotic surgeries (TORS) in order to render patients free of gross disease. Adjuvant radiotherapy, when indicated, is then often reduced in total dose, and the need 

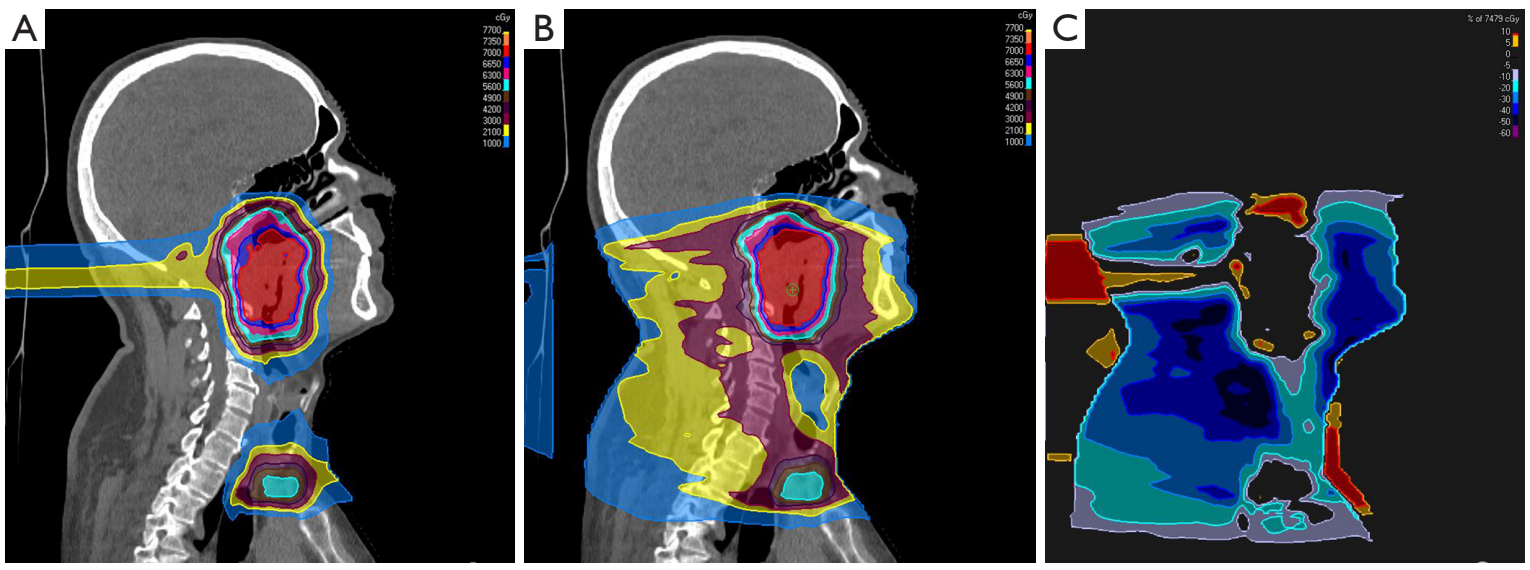

Figure 2 Comparison of pencil beam scanning proton therapy plan (A) versus volumetric modulated arc therapy plan (B) for patient with head and neck squamous cell carcinoma of the right tonsil. A subtraction dose (C) is also provided to show areas in which the proton plan reduces dose (cool/blue) or increases dose (red/hot). Note the substantial reductions in dose to the oral cavity and midline pharyngeal/ laryngeal structures.

for concurrent chemotherapy is frequently obviated. The recent publication of the ORATOR study, which was a multicenter, international, randomized, phase II study, may temper excitement for this strategy as it showed relative equivalence in outcomes between definitive chemoradiotherapy and primary TORS with adjuvant therapy in patients with OPSCC (54). However, the sample size $(n=68)$ and the slight favoring of chemoradiotherapy in MDADI scores may have raised more questions than answers. Additionally, the Eastern Cooperative Oncology Group (ECOG) has yet to report results from ECOG 3311 which was a prospective study tailored to assessing outcomes following the primary surgical, TORS approach. These results are eagerly awaited.

While salivary gland transfer procedures and radiation cytoprotectants/mitigators have been attempted with some success, neither has become a widely utilized standard. Salivary gland transfers involve transposition of the submandibular gland, especially to the submental space during surgical intervention. This displaces the gland from the high-dose, adjuvant radiotherapy region. Several small series have shown promise, but the rates of over-treatment (patients undergoing transfer without adjuvant radiotherapy) and the required surgical expertise have deterred widespread implementation (55-57). Radioprotectants, such as Amifostine, have gained traction in the radiotherapy community based on favorable clinical trial results; however, concerns regarding tumoral protection or drug-associated side effects have, again, limited use of these strategy $(58,59)$.
While prevention remains the preferred strategy for reducing the impact of radiation-associated xerostomia in patients with HNSCC, some level of xerostomia is essentially inevitable in patients undergoing this oncologic approach. Therefore, treatment regimens to lessen the impact or symptoms associated with xerostomia are also of great importance.

\section{Techniques for palliation and mitigation of radiation-induced xerostomia}

When xerostomia occurs in patients undergoing or who have undergone radiotherapy for HNSCC, two approaches for mitigation of symptomatology are most commonly utilized-replacement with salivary substitutes and stimulation with oral or peripheral agents.

Intraoral substitutes are widely commercially available without a prescription both for oncologic and nononcologic patients with xerostomia. These are notorious for providing some relief that is unfortunately short-lived for patients suffering from this sequela. A recent systematic review excellently summarized the available agents and their mechanisms (60). Several studies have centered around various salivary substitutes and either head-to-head comparison or their effectiveness versus water alone (61). Unfortunately, many of these studies remain flawed in design, lacking in objective clinical data, or ambiguous as to a preferred strategy. In fact, some have found frequent sips of water to be nearly or just as effective (62). Mucoadhesive 
discs of xylitol or sugar free gums containing the same have been utilized with mixed results as well (63).

One of the key criticisms of this approach is that most salivary substitutes lack some of the essential functions of saliva. They fail to provide the antimicrobial and immunologic functions of normal saliva, and therefore, antimicrobial rinses and/or stringent oral hygiene agents must be added to achieve similar protection $(64,65)$. In practice, most patients are also given a prescription-level fluoride toothpaste with normally four times the level of fluoride for cariogenic protection.

Pilocarpine has been attempted as a "topical" mouth rinse/spray, lozenges, or systemic tablets in patients with dry mouth syndromes such as Sjogren's or in patients post-radiotherapy. Multiple trials have compared these interventions to no treatment, placebo, or each other. Again, none have presented convincing results of dramatic improvements in outcomes (66-69). Clinicians may attempt these with patients and continue if subjective improvements, but especially with systemic administration, these therapies can come with side effects. Also, it is unclear the level of systemic absorption transmucosal or when the rinses are swallowed. These factors may make ineligible a large number of patients with asthma, glaucoma, cardiovascular disease, or respiratory illnesses like chronic obstructive pulmonary disease.

Cevimeline is a parasympathomimetic/muscarinic agonist that has demonstrated efficacy in Sjogren's syndrome. This has also been tested in the setting of radiation-induced xerostomia in at least two sizeable studies $(70,71)$. While there was a signal for improvement of unclear magnitude, the sweating and gastrointestinal side effects were substantial deterrents to patients' continuation of therapy. Again, contraindications to this therapy may further challenge implementation.

Acupuncture is also a frequently implemented palliative therapy for xerostomia. Three studies have attempted to clarify its role and benefits (72-74). Perhaps most interestingly, one of these attempted sham acupuncture versus targeted but failed to show a substantial improvement (74). Nevertheless, this intervention comes with relatively limited side effects and potentially other unrelated benefits.

In summary, numerous ameliorative, palliative approaches exist for radiation-induced xerostomia. These efforts continue to blur the lines between supportive care and early palliative care, but regardless of the terminology, attention to these symptoms and prompt treatment remain critically important and meaningful to a patient's quality of life (75). While none are ubiquitously effective and many come with limitations or side effects, several are worthwhile in attempting with patients who are particularly impacted by their xerostomia. This becomes somewhat of a trial and error approach for the clinician and patient, but often, patients experience at least partial relief with one of these strategies.

\section{Conclusions}

Radiation-induced xerostomia, especially in the treatment of HNSCC, remains one of the most common and impactful toxicities associated with oncologic therapy in this patient population. With improvements in curative therapies overall and a shift in etiology of this disease towards HPV-OPSCC, a growing number of patients are surviving to experience this morbidity long-term. Technological and therapeutic advancements in radiotherapy delivery for xerostomia prevention are promising and currently being implemented or investigated. Palliative measures for mitigating the impact of radiation-induced xerostomia are numerous and varied. Finding the right fit for individual patients remains somewhat trial and error. Further research is warranted to clarify comparative effectiveness and reproducibility of results.

\section{Acknowledgments}

Funding: None.

\section{Footnote}

Provenance and Peer Review: This article was commissioned by the Guest Editors (Jan Gaertner, Charles B. Simone II and Fiona Lim) for the series "Clinical Challenges and Pitfalls in Early Palliative Care: Practical Knowledge and Guidance from other Medical Specialties" published in Annals of Palliative Medicine. The article was sent for external peer review organized by the Guest Editors and the editorial office.

Conflicts of Interest: Both authors have completed the ICMJE uniform disclosure form (available at http://dx.doi. org/10.21037/apm.2020.02.36). The series "Clinical Challenges and Pitfalls in Early Palliative Care: Practical Knowledge and Guidance from other Medical Specialties" was commissioned by the editorial office without any funding or sponsorship. Dr. Snider declares: Varian Medical 
Systems Honorarium, UM Ventures grant for novel mucositis mitigation device development, MIPS grant for GammaPod device development, Patent pending for novel proton therapy technique, Siemens Healthineers consultant funding, travel grants from Pyrexar/STM, Hefei Ion Center Symposium, Moscow ONE Symposium, CSCO Varian, PTCOG Varian. None of these have substantially biased the work or opinions, herein. The authors have no other conflicts of interest to declare.

Open Access Statement: This is an Open Access article distributed in accordance with the Creative Commons Attribution-NonCommercial-NoDerivs 4.0 International License (CC BY-NC-ND 4.0), which permits the noncommercial replication and distribution of the article with the strict proviso that no changes or edits are made and the original work is properly cited (including links to both the formal publication through the relevant DOI and the license). See: https://creativecommons.org/licenses/by-nc-nd/4.0/.

\section{References}

1. Siegel RL, Miller KD, Jemal A. Cancer statistics, 2019. CA Cancer J Clin 2019;69:7-34.

2. National Program of Cancer Registries SEER*Stat Database: U.S. Cancer Statistics Incidence Analytic file 1998-2016. United States Department of Health and Human Services, Centers for Disease Control and Prevention. Released June 2019, based on the November 2018 submission.

3. Ferlay J, Soerjomataram I, Dikshit R, et al. Cancer incidence and mortality worldwide: sources, methods and major patterns in GLOBOCAN 2012. Int J Cancer 2015;136:E359-86.

4. American Cancer Society. Cancer Facts \& Figures 2019. Atlanta: American Cancer Society; 2019.

5. Ang KK, Harris J, Wheeler R, et al. Human papillomavirus and survival of patients with oropharyngeal cancer. N Engl J Med 2010;363:24-35.

6. Viens LJ, Henley SJ, Watson M, et al. Human papillomavirus-associated cancers - United States, 20082012. MMWR Morb Mortal Wkly Rep 2016;65:661-6.

7. Adelstein D, Gillison ML, Pfister DG, et al. NCCN Guidelines Insights: Head and Neck Cancers, Version 2.2017. J Natl Compr Canc Netw 2017;15:761-70.

8. Strojan P, Hutcheson KA, Eisbruch A, et al. Treatment of late sequelae after radiotherapy for head and neck cancer. Cancer Treat Rev 2017;59:79-92.
9. Humphrey SP, Williamson RT. A review of saliva: normal composition, flow, and function. J Prosthet Dent 2001;85:162-9.

10. Grégoire V, Ang K, Budach W, et al. Delineation of the neck node levels for head and neck tumors: a 2013 update. DAHANCA, EORTC, HKNPCSG, NCIC CTG, NCRI, RTOG, TROG consensus guidelines. Radiother Oncol 2014;110:172-81.

11. Dirix P, Nuyts S, Van den Bogaert W. Radiation-induced xerostomia in patients with head and neck cancer: a literature review. Cancer 2006;107:2525-34.

12. Konings AW, Coppes RP, Vissink A. On the mechanism of salivary gland radiosensitivity. Int J Radiat Oncol Biol Phys 2005;62:1187-94.

13. Roesink JM, Moerland MA, Battermann JJ, et al. Quantitative dose-volume response analysis of changes in parotid gland function after radiotherapy in the head-andneck region. Int J Radiat Oncol Biol Phys 2001;51:938-46.

14. Eneroth CM, Henrikson CO, Jakobsson PA.. Effect of fractionated radiotherapy on salivary gland function. Cancer 1972;30:1147-53.

15. Valdez IH. Radiation-induced salivary dysfunction: clinical course and significance. Spec Care Dentist 1991;11:252-5.

16. PDQ Supportive and Palliative Care Editorial Board. PDQ Oral Complications of Chemotherapy and Head/ Neck Radiation. Bethesda, MD: National Cancer Institute. Updated 12/16/2016. Available online: https://www.cancer. gov/about-cancer/treatment/side-effects/mouth-throat/ oral-complications-hp-pdq. Accessed 06/01/2019.

17. Jensen SB, Pedersen AM, Vissink A, et al. A systematic review of salivary gland hypofunction and xerostomia induced by cancer therapies: prevalence, severity and impact on quality of life. Support Care Cancer 2010;18:1039-60.

18. Buglione M, Cavagnini R, Di Rosario F, et al. Oral toxicity management in head and neck cancer patients treated with chemotherapy and radiation: Xerostomia and trismus (Part 2). Literature review and consensus statement. Crit Rev Oncol Hematol 2016;102:47-54.

19. Brown LR, Dreizen S, Handler S, et al. Effect of radiationinduced xerostomia on human oral microflora. J Dent Res 1975;54:740-50.

20. Valdez IH, Atkinson JC, Ship JA, et al. Major salivary gland function in patients with radiation-induced xerostomia: flow rates and sialochemistry. Int J Radiat Oncol Biol Phys 1993;25:41-7.

21. Eisbruch A, Ten Haken RK, Kim HM, et al. Dose, volume, and function relationships in parotid salivary glands 
following conformal and intensity-modulated irradiation of head and neck cancer. Int J Radiat Oncol Biol Phys 1999;45:577-87.

22. Vissink A, Jansma J, Spijkervet FK, et al. Oral sequelae of head and neck radiotherapy. Crit Rev Oral Biol Med 2003;14:199-212.

23. Hunter KU, Schipper M, Feng FY, et al. Toxicities affecting quality of life after chemo-IMRT of oropharyngeal cancer: prospective study of patientreported, observer-rated, and objective outcomes. Int J Radiat Oncol Biol Phys 2013;85:935-40.

24. Oates J, Davies S, Roydhouse JK, et al. The effect of cancer stage and treatment modality on quality of life in oropharyngeal cancer. Laryngoscope 2014;124:151-8.

25. Vainshtein JM, Moon DH, Feng FY, et al. Long-term quality of life after swallowing and salivary-sparing chemointensity modulated radiation therapy in survivors of human papillomavirus-related oropharyngeal cancer. Int J Radiat Oncol Biol Phys 2015;91:925-33.

26. Al-Mamgani A, van Rooij P, Tans L, et al. Prospective evaluation of patient reported quality-of-life after (chemo) radiation for oropharyngeal cancer: which patients are at risk of significant quality-of-life deterioration? Radiother Oncol 2013;106:359-63.

27. Ogama N, Suzuki S. Adverse effects and appetite suppression associated with particle beam therapy in patients with head and neck cancer. Jpn J Nurs Sci 2012;9:28-37.

28. Ogama N, Suzuki S, Umeshita K, et al. Appetite and adverse effects associated with radiation therapy in patients with head and neck cancer. Eur J Oncol Nurs 2010;14:3-10.

29. Langius JA, Doornaert P, Spreeuwenberg MD, et al. Radiotherapy on the neck nodes predicts severe weight loss in patients with early stage laryngeal cancer. Radiother Oncol 2010;97:80-5.

30. Kubrak C, Olson K, Jha N, et al. Clinical determinants of weight loss in patients receiving radiation and chemoirradiation for head and neck cancer: a prospective longitudinal view. Head Neck 2013;35:695-703.

31. Kubrak C, Olson K, Jha N, et al. Nutrition impact symptoms: key determinants of reduced dietary intake, weight loss, and reduced functional capacity of patients with head and neck cancer before treatment. Head Neck 2010;32:290-300.

32. Beetz I, Burlage FR, Bijl HP, et al. The Groningen Radiotherapy-Induced Xerostomia questionnaire: development and validation of a new questionnaire.
Radiother Oncol 2010;97:127-31.

33. Langendijk JA, Doornaert P, Verdonck-de Leeuw IM, et al. Impact of late treatment-related toxicity on quality of life among patients with head and neck cancer treated with radiotherapy. J Clin Oncol 2008;26:3770-6.

34. Høxbroe Michaelsen S, Grønhøj C, Høxbroe Michaelsen J, et al. Quality of life in survivors of oropharyngeal cancer: A systematic review and meta-analysis of 1366 patients. Eur J Cancer 2017;78:91-102.

35. Li Y, Taylor JM, Ten Haken RK, et al. The impact of dose on parotid salivary recovery in head and neck cancer patients treated with radiation therapy. Int J Radiat Oncol Biol Phys 2007;67:660-9.

36. Blanco AI, Chao KS, El Naqa I, et al. Dose-volume modeling of salivary function in patients with head-andneck cancer receiving radiotherapy. Int J Radiat Oncol Biol Phys 2005;62:1055-69.

37. Roesink JM, Schipper M, Busschers W, et al. A comparison of mean parotid gland dose with measures of parotid gland function after radiotherapy for head-and-neck cancer: implications for future trials. Int J Radiat Oncol Biol Phys 2005;63:1006-9.

38. Braam PM, Roesink JM, Moerland MA, et al. Long-term parotid gland function after radiotherapy. Int J Radiat Oncol Biol Phys 2005;62:659-64.

39. Murdoch-Kinch CA, Kim HM, Vineberg KA, et al. Dose-effect relationships for the submandibular salivary glands and implications for their sparing by intensity modulated radiotherapy. Int J Radiat Oncol Biol Phys 2008;72:373-82.

40. Pow EH, Kwong DL, McMillan AS, et al. Xerostomia and quality of life after intensity-modulated radiotherapy vs. conventional radiotherapy for early-stage nasopharyngeal carcinoma: initial report on a randomized controlled clinical trial. Int J Radiat Oncol Biol Phys 2006;66:981-91.

41. Kam MK, Leung SF, Zee B, et al. Prospective randomized study of intensity-modulated radiotherapy on salivary gland function in early-stage nasopharyngeal carcinoma patients. J Clin Oncol 2007;25:4873-9.

42. Nutting CM, Morden JP, Harrington KJ, et al. Parotidsparing intensity modulated versus conventional radiotherapy in head and neck cancer (PARSPORT): a phase 3 multicentre randomised controlled trial. Lancet Oncol 2011;12:127-36.

43. Grégoire V, Evans M, Le QT, et al. Delineation of the primary tumour Clinical Target Volumes (CTV-P) in laryngeal, hypopharyngeal, oropharyngeal and oral cavity squamous cell carcinoma: AIRO, CACA, DAHANCA, 
EORTC, GEORCC, GORTEC, HKNPCSG, HNCIG, IAG-KHT, LPRHHT, NCIC CTG, NCRI, NRG Oncology, PHNS, SBRT, SOMERA, SRO, SSHNO, TROG consensus guidelines. Radiother Oncol 2018;126:3-24.

44. Brouwer CL, Steenbakkers RJ, Bourhis J, et al. CT-based delineation of organs at risk in the head and neck region: DAHANCA, EORTC, GORTEC, HKNPCSG, NCIC CTG, NCRI, NRG Oncology and TROG consensus guidelines. Radiother Oncol 2015;117:83-90.

45. Lee NCJ, Kelly JR, Park HS, et al. The risk of level IB nodal involvement in oropharynx cancer: Guidance for submandibular gland sparing irradiation. Pract Radiat Oncol 2017;7:e317-21.

46. Lee $\mathrm{AW}, \mathrm{Ng} W \mathrm{~T}$, Pan JJ, et al. International guideline for the delineation of the clinical target volumes (CTV) for nasopharyngeal carcinoma. Radiother Oncol 2018;126:25-36.

47. Jackson WC, Hawkins PG, Arnould GS, et al. Submandibular gland sparing when irradiating neck level IB in the treatment of oral squamous cell carcinoma. Med Dosim 2019;44:144-9.

48. Huang SH, Waldron J, Bratman SV, et al. Re-evaluation of Ipsilateral Radiation for T1-T2N0-N2b Tonsil Carcinoma at the Princess Margaret Hospital in the Human Papillomavirus Era, 25 Years Later. Int J Radiat Oncol Biol Phys 2017;98:159-69.

49. O'Sullivan B, Warde P, Grice B, et al. The benefits and pitfalls of ipsilateral radiotherapy in carcinoma of the tonsillar region. Int J Radiat Oncol Biol Phys 2001;51:332-43.

50. Holliday EB, Kocak-Uzel E, Feng L, et al. Dosimetric advantages of intensity-modulated proton therapy for oropharyngeal cancer compared with intensity-modulated radiation: A case-matched control analysis. Med Dosim 2016;41:189-94.

51. Blanchard P, Garden AS, Gunn GB, et al. Intensitymodulated proton beam therapy (IMPT) versus intensitymodulated photon therapy (IMRT) for patients with oropharynx cancer: A case-matched analysis. Radiother Oncol 2016;120:48-55.

52. Sio TT, Lin HK, Shi Q, et al. Intensity-modulated proton therapy versus intensity-modulated photon radiation therapy for oropharyngeal cancer: First comparative results of patient-reported outcomes. Int J Radiat Oncol Biol Phys 2016;95:1107-14.

53. Chera BS, Amdur RJ, Tepper JE, et al. Mature results of a prospective study of deintensified chemoradiotherapy for low-risk human papillomavirus-associated oropharyngeal squamous cell carcinoma. Cancer 2018;124:2347-54.

54. Nichols AC, Theurer J, Prisman E, et al. Radiotherapy versus transoral robotic surgery and neck dissection for oropharyngeal squamous cell carcinoma (ORATOR): an open-label, phase 2, randomised trial. Lancet Oncol 2019;20:1349-59.

55. Jha N, Seikaly H, Harris J, et al. Prevention of radiation induced xerostomia by surgical transfer of submandibular salivary gland into the submental space. Radiother Oncol 2003;66:283-9.

56. Jha N, Seikaly H, McGaw T, et al. Submandibular salivary gland transfer prevents radiation-induced xerostomia. Int J Radiat Oncol Biol Phys 2000;46:7-11.

57. Seikaly H, Jha N, McGaw T, et al. Submandibular gland transfer: a new method of preventing radiation-induced xerostomia. Laryngoscope 2001;111:347-52.

58. Wasserman TH, Brizel DM, Henke M, et al. Influence of intravenous amifostine on xerostomia, tumor control and survival after radiotherapy for head-and-neck cancer: a 2-year followup of a prospective, randomized, phase III trial. Int J Radiat Oncol Biol Phys 2005;63:985-90.

59. Brizel DM, Wasserman TH, Henke M, et al. Phase III randomized trial of amifostine as a radioprotector in head and neck cancer. J Clin Oncol 2000;18:3339-45.

60. Jensen SB, Pedersen AM, Vissink A, et al. A systematic review of salivary gland hypofunction and xerostomia induced by cancer therapies: management strategies and economic impact. Support Care Cancer 2010;18:1061-79.

61. Momm F, Volegova-Neher NJ, Schulte-Mönting J, et al. Different saliva substitutes for treatment of xerostomia following radiotherapy a prospective crossover study. Strahlenther Onkol 2005;181:231-6.

62. Jellema AP, Langendijk H, Bergenhenegouwen L, et al. The efficacy of Xialine in patients with xerostomia resulting from radiotherapy for head and neck cancer. Radiother Oncol 2001;59:157-60.

63. Kerr AR, Corby PM, Shah SS, et al. Use of a mucoadhesive disk for relief of dry mouth: a randomized, double-masked, controlled crossover study. J Am Dent Assoc 2010;141:1250-6.

64. Epstein JB, McBride B, Stevenson-Moore P, et al. The efficacy of chlorhexidine gel in reduction of Streptococcus mutans and Lactobacillus species in patients treated with radiation therapy. Oral Surg Oral Med Oral Pathol 1991;71:172-8.

65. Epstein JB, Loh R, Stevenson-Moore P, et al. Chlorhexidine rinse in prevention of dental caries in 
patients following radiation therapy. Oral Surg Oral Med Oral Pathol 1989;68:401-5.

66. Davies AN, Singer J. A comparison of artificial saliva and pilocarpine in radiation-induced xerostomia. J Laryngol Otol 1994;108:663-5.

67. LeVeque FG, Montgomery M, Potter D, et al. A multicenter, randomized, double-blind, placebocontrolled, dose-titration study of oral pilocarpine for treatment of radiation-induced xerostomia in head and neck cancer patients. J Clin Oncol 1993;11:1124-31.

68. Johnson JT, Ferretti GA, Nethery WJ, et al. Oral pilocarpine for post-irradiation xerostomia in patients with head and neck cancer. N Engl J Med 1993;329:390-5.

69. Taweechaisupapong S, Pesee M, Aromdee C, et al. Efficacy of pilocarpine lozenge for post-radiation xerostomia in patients with head and neck cancer. Aust Dent J 2006;51:333-7.

70. Chambers MS, Posner M, Jones CU, et al. Cevimeline for the treatment of postirradiation xerostomia in patients with head and neck cancer. Int J Radiat Oncol Biol Phys 2007;68:1102-9.

71. Witsell DL, Stinnett S, Chambers MS. Effectiveness of cevimeline to improve oral health in patients with postradiation xerostomia. Head Neck 2012;34:1136-42.

72. Blom M, Dawidson I, Fernberg JO, et al. Acupuncture treatment of patients with radiation-induced xerostomia. Eur J Cancer B Oral Oncol 1996;32B:182-90.

73. Simcock R, Fallowfield L, Monson K, et al. Arix: a randomised trial of acupuncture $\mathrm{v}$ oral care sessions in patients with chronic xerostomia following treatment of head and neck cancer. Ann Oncol 2013;24:776-83.

74. Cho JH, Chung WK, Kang W, et al. Manual acupuncture improved quality of life in cancer patients with radiationinduced xerostomia. J Altern Complement Med 2008;14:523-6.

75. Gaertner J, Lutz S, Chow E, et al. Integrating palliative care and oncology: towards a common understanding. Ann Palliat Med 2015;4:3-4.

Cite this article as: Snider JW 3rd, Paine CC 2nd. Sticky stuff: xerostomia in patients undergoing head and neck radiotherapy - prevalence, prevention, and palliative care. Ann Palliat Med 2020;9(3):1340-1350. doi: 10.21037/apm.2020.02.36 